Supplementary Information

\title{
Unexpected polymorphism in bromoantimonate(III) complexes and its impact on optical properties
}

Andrey N. Usoltsev, ${ }^{1}$ Taisiya S. Sukhikh, ${ }^{1}$ Alexander S. Novikov, ${ }^{2}$ Vladimir R. Shayapov, ${ }^{1}$ Denis P. Pishchur, ${ }^{1}$ Ilya V. Korolkov, ${ }^{1}$ Ilyas F. Sakhapov, ${ }^{3}$ Vladimir P. Fedin, ${ }^{1}$ Maxim N. Sokolov ${ }^{1}$ and Sergey A. Adonin ${ }^{1,3 *}$

${ }^{1}$ Nikolaev Institute of Inorganic Chemistry SB RAS, 630090 Lavrentieva St., Novosibirsk, Russia. E-mail: adonin@niic.nsc.ru

${ }^{2}$ Saint Petersburg State University, 199034 Universitetskaya Nab., 7/9, Saint Petersburg, Russia.

${ }^{3}$ South Ural State University, 454080 Lenina St. 76, Chelyabinsk, Russia.

Table S1. Crystal data and structure refinement for compounds 1-5.

\begin{tabular}{|c|c|c|c|c|c|c|}
\hline Identification code & 1 & 2 & 3 & $4(298 K)$ & $4(150 \mathrm{~K})$ & 5 \\
\hline Empirical formula & $\mathrm{C}_{12} \mathrm{H}_{14} \mathrm{Br}_{5} \mathrm{~N}_{2} \mathrm{Sb}$ & $\mathrm{C}_{16} \mathrm{H}_{22} \mathrm{Br}_{5} \mathrm{~N}_{2} \mathrm{Sb}$ & $\mathrm{C}_{24} \mathrm{H}_{28} \mathrm{Br}_{10} \mathrm{~N}_{4} \mathrm{Sb}_{2}$ & $\mathrm{C}_{24} \mathrm{H}_{28} \mathrm{Br}_{10} \mathrm{~N}_{4} \mathrm{Sb}_{2}$ & $\mathrm{C}_{24} \mathrm{H}_{28} \mathrm{Br}_{10} \mathrm{~N}_{4} \mathrm{Sb}_{2}$ & $\mathrm{C}_{16} \mathrm{H}_{22} \mathrm{Br}_{8} \mathrm{~N}_{2} \mathrm{Sb}_{2}$ \\
\hline Formula weight & 707.55 & 763.65 & 1415.10 & 1415.10 & 1415.10 & 1125.13 \\
\hline Temperature/K & $140(2)$ & $150(2)$ & $130(2)$ & $298(2)$ & $150(2)$ & $130(2)$ \\
\hline Space group & $P-1$ & $P 2 / n$ & $P-1$ & $C 2 / c$ & $C 2 / c$ & $P 2_{1} / n$ \\
\hline$a / \AA ̊$ & $6.1343(3)$ & $6.2898(4)$ & $10.1060(4)$ & $19.6880(8)$ & $19.6628(8)$ & $20.5536(8)$ \\
\hline $\mathrm{b} / \AA ̊ \AA$ & $9.2857(4)$ & $8.7454(7)$ & $10.2956(4)$ & $10.0152(5)$ & $9.9517(4)$ & $7.2974(2)$ \\
\hline$c / A ̊$ & $17.2041(9)$ & $20.4076(16)$ & $11.1394(4)$ & $20.8410(9)$ & $20.6906(8)$ & $21.2510(7)$ \\
\hline$\alpha /^{\circ}$ & $90.331(4)$ & 90 & $105.030(3)$ & 90 & 90 & 90 \\
\hline$\beta /{ }^{\circ}$ & $100.259(4)$ & $90.438(3)$ & 101.709(3) & $111.104(2)$ & $111.3160(10)$ & $117.694(5)$ \\
\hline$\gamma /{ }^{\circ}$ & $99.912(4)$ & 90 & $117.233(4)$ & 90 & 90 & 90 \\
\hline Volume $/ \AA^{3}$ & $949.24(8)$ & $1122.52(14)$ & $922.86(7)$ & $3833.8(3)$ & $3771.7(3)$ & $2822.3(2)$ \\
\hline z & 2 & 2 & 1 & 4 & 4 & 4 \\
\hline$\rho_{\text {calc }} \mathrm{g} / \mathrm{cm}^{3}$ & 2.476 & 2.259 & 2.546 & 2.452 & 2.492 & 2.648 \\
\hline$\mu / \mathrm{mm}^{-1}$ & 11.969 & 10.130 & 12.311 & 11.854 & 12.049 & 13.249 \\
\hline$F(000)$ & 652.0 & 716.0 & 652.0 & 2608.0 & 2608.0 & 2056.0 \\
\hline Crystal size $/ \mathrm{mm}^{3}$ & $0.15 \times 0.15 \times 0.1$ & $0.1 \times 0.08 \times 0.04$ & $\begin{array}{c}0.270 \times 0.170 \times \\
0.140\end{array}$ & $0.38 \times 0.2 \times 0.15$ & $0.12 \times 0.08 \times 0.06$ & $\begin{array}{c}0.280 \times 0.170 \times \\
0.160\end{array}$ \\
\hline Radiation & $\begin{array}{c}\operatorname{MoK} \alpha(\lambda= \\
0.71073)\end{array}$ & $\begin{array}{c}\operatorname{MoK} \alpha(\lambda= \\
0.71073)\end{array}$ & $\begin{array}{c}\operatorname{MoK} \alpha(\lambda= \\
0.71073)\end{array}$ & $\begin{array}{c}\operatorname{MoK} \alpha(\lambda= \\
0.71073)\end{array}$ & $\begin{array}{c}\operatorname{MoK} \alpha(\lambda= \\
0.71073)\end{array}$ & $\begin{array}{c}\operatorname{MoK} \alpha(\lambda= \\
0.71073)\end{array}$ \\
\hline $2 \Theta$ range for data collection $/^{\circ}$ & 4.456 to 57.92 & 4.658 to 52.98 & 7.454 to 57.8 & 4.436 to 57.394 & 4.226 to 61.162 & 6.736 to 57.748 \\
\hline Index ranges & $\begin{array}{c}-8 \leq h \leq 7,-11 \leq k \\
\leq 9,-21 \leq 1 \leq 22\end{array}$ & $\begin{array}{l}-7 \leq h \leq 7,-10 \leq k \\
\leq 10,-25 \leq 1 \leq 25\end{array}$ & $\begin{array}{l}-12 \leq h \leq 11,-13 \leq \\
k \leq 12,-12 \leq I \leq 13\end{array}$ & $\begin{array}{l}-26 \leq h \leq 26,-13 \leq \\
k \leq 13,-28 \leq 1 \leq 28\end{array}$ & $\begin{array}{l}-28 \leq h \leq 24,-13 \leq \\
k \leq 14,-29 \leq 1 \leq 29\end{array}$ & $\begin{array}{c}-27 \leq h \leq 20,-8 \leq k \\
\leq 9,-15 \leq 1 \leq 25\end{array}$ \\
\hline Reflections collected & 7530 & 14998 & 6813 & 24806 & 18242 & 13283 \\
\hline Independent reflections & $\begin{array}{c}4166\left[R_{\text {int }}=\right. \\
0.0212, R_{\text {sigma }}= \\
0.0365]\end{array}$ & $\begin{array}{c}2289\left[R_{\text {int }}=\right. \\
0.0478, R_{\text {sigma }}= \\
0.0332]\end{array}$ & $\begin{array}{c}3979\left[R_{\text {int }}=\right. \\
0.0261, R_{\text {sigma }}= \\
0.0496]\end{array}$ & $\begin{array}{c}4944\left[R_{\text {int }}=\right. \\
0.0363, R_{\text {sigma }}= \\
0.0282]\end{array}$ & $\begin{array}{c}5474\left[R_{\text {int }}=\right. \\
0.0342, R_{\text {sigma }}= \\
0.0375]\end{array}$ & $\begin{array}{c}6039\left[R_{\text {int }}=\right. \\
0.0238, R_{\text {sigma }}= \\
0.0355]\end{array}$ \\
\hline Restraints/parameters & $0 / 181$ & $48 / 117$ & $0 / 181$ & $0 / 182$ & $0 / 182$ & $0 / 253$ \\
\hline Goodness-of-fit on $F^{2}$ & 1.020 & 1.403 & 1.037 & 1.159 & 1.026 & 1.114 \\
\hline Final R indexes $[1>=2 \sigma(I)]$ & $\begin{aligned} \mathrm{R}_{1} & =0.0270 \\
\mathrm{wR}_{2} & =0.0543\end{aligned}$ & $\begin{aligned} \mathrm{R}_{1} & =0.0971 \\
\mathrm{wR}_{2} & =0.2403\end{aligned}$ & $\begin{aligned} \mathrm{R}_{1} & =0.0334 \\
\mathrm{wR}_{2} & =0.0689\end{aligned}$ & $\begin{aligned} \mathrm{R}_{1} & =0.0284 \\
\mathrm{wR}_{2} & =0.0636\end{aligned}$ & $\begin{aligned} \mathrm{R}_{1} & =0.0221 \\
\mathrm{wR}_{2} & =0.0436\end{aligned}$ & $\begin{aligned} R_{1} & =0.0265 \\
w R_{2} & =0.0501\end{aligned}$ \\
\hline Final $R$ indexes [all data] & $\begin{aligned} R_{1} & =0.0353 \\
w R_{2} & =0.0578\end{aligned}$ & $\begin{array}{l}\mathrm{R}_{1}=0.1000 \\
\mathrm{wR}_{2}=0.2421\end{array}$ & $\begin{aligned} \mathrm{R}_{1} & =0.0419 \\
\mathrm{wR}_{2} & =0.0730\end{aligned}$ & $\begin{aligned} R_{1} & =0.0346 \\
w_{2} & =0.0651\end{aligned}$ & $\begin{aligned} \mathrm{R}_{1} & =0.0279 \\
\mathrm{wR}_{2} & =0.0451\end{aligned}$ & $\begin{array}{l}\mathrm{R}_{1}=0.0367 \\
\mathrm{wR}_{2}=0.0526\end{array}$ \\
\hline Largest diff. peak/hole / e $\AA^{-3}$ & $0.69 /-1.11$ & $3.45 /-3.26$ & $0.95 /-1.69$ & $0.58 /-1.63$ & $0.54 /-0.73$ & $0.62 /-0.84$ \\
\hline
\end{tabular}


Table S2. Sb-Br bond lengths (Å) for compounds 1-5.

\begin{tabular}{|c|c|c|c|c|c|c|c|c|c|c|c|}
\hline \multicolumn{2}{|c|}{1} & \multicolumn{2}{|c|}{2} & \multicolumn{2}{|c|}{3} & \multicolumn{2}{|c|}{$4(298 K)$} & \multicolumn{2}{|c|}{$4(150 \mathrm{~K})$} & \multicolumn{2}{|c|}{5} \\
\hline $\mathrm{Sb} 1-\mathrm{Br} 1$ & $2.7497(4)$ & $\mathrm{Sb} 1-\mathrm{Br} 1$ & $2.722(3)$ & $\mathrm{Sb} 1-\mathrm{Br} 1$ & $2.7219(5)$ & $\mathrm{Sb} 1-\mathrm{Br} 1$ & $2.7828(3)$ & $\mathrm{Sb} 1-\mathrm{Br} 1$ & $2.7808(3)$ & $\mathrm{Sb} 1-\mathrm{Br} 1$ & $2.8935(4)$ \\
\hline $\mathrm{Sb} 1-\mathrm{Br} 2$ & $2.7426(4)$ & Sb1-Br11 & $2.722(3)$ & $\mathrm{Sb} 1-\mathrm{Br} 2$ & $2.8722(6)$ & $\mathrm{Sb} 1-\mathrm{Br} 2$ & $2.6620(4)$ & $\mathrm{Sb} 1-\mathrm{Br} 2$ & $2.6627(2)$ & $\mathrm{Sb} 1^{3}-\mathrm{Br} 1$ & $3.0060(5)$ \\
\hline $\mathrm{Sb} 1-\mathrm{Br} 5$ & $2.8379(4)$ & $\mathrm{Sb} 1-\mathrm{Br} 2$ & $2.813(3)$ & $\mathrm{Sb} 1-\mathrm{Br} 3$ & $2.8552(5)$ & $\mathrm{Sb} 1-\mathrm{Br} 3$ & $2.6295(3)$ & $\mathrm{Sb} 1-\mathrm{Br} 3$ & $2.6317(2)$ & $\mathrm{Sb} 1-\mathrm{Br} 2$ & $2.6223(5)$ \\
\hline $\mathrm{Sb} 1-\mathrm{Br} 3$ & $2.5468(4)$ & $\mathrm{Sb} 1-\mathrm{Br} 3$ & $2.580(4)$ & $\mathrm{Sb} 1-\mathrm{Br} 5$ & $2.7463(6)$ & $\mathrm{Sb} 1^{2}-\mathrm{Br} 5$ & $3.0242(4)$ & $\mathrm{Sb} 1^{2}-\mathrm{Br} 5$ & $3.0190(3)$ & $\mathrm{Sb} 1-\mathrm{Br} 4$ & $2.7269(4)$ \\
\hline & & & & & & $\mathrm{Sb} 1-\mathrm{Br} 5$ & $3.0242(4)$ & $\mathrm{Sb} 1-\mathrm{Br} 5$ & $3.0190(3)$ & $\mathrm{Sb} 2-\mathrm{Br} 5$ & $2.6513(5)$ \\
\hline & & & & & & & & & & $\mathrm{Sb} 2-\mathrm{Br} 6$ & $2.9480(5)$ \\
\hline & & & & & & & & & & $\mathrm{Sb} 2-\mathrm{Br} 8$ & $2.5539(5)$ \\
\hline & & & & & & & & & & $\mathrm{Br} 1^{3}-\mathrm{Sb} 1$ & $3.0060(5)$ \\
\hline & & & & & & & & & & $\mathrm{Br}^{4}-\mathrm{Sb} 2$ & $2.9783(5)$ \\
\hline
\end{tabular}

${ }^{1} 1 / 2-X,+Y, 1 / 2-Z ; 1 / 2-Z ;{ }^{2} 2-X,+Y, 1 / 2-Z ;{ }^{3} 1-X,-Y, 1-Z ;{ }^{4}-X, 1-Y, 1-Z$

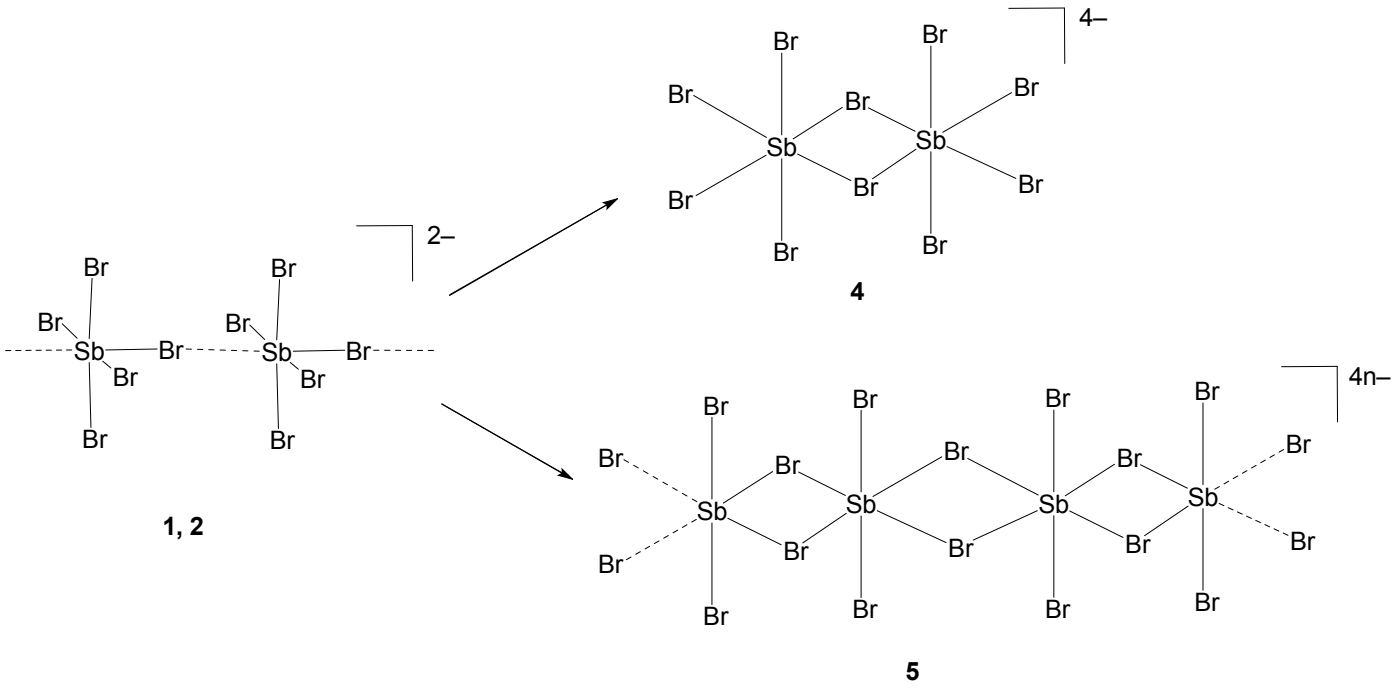

Scheme S1. Transformation of $\mathbf{1}$ and $\mathbf{2}$ to $\mathbf{4}$ and 5, respectively. 


\section{S-3}

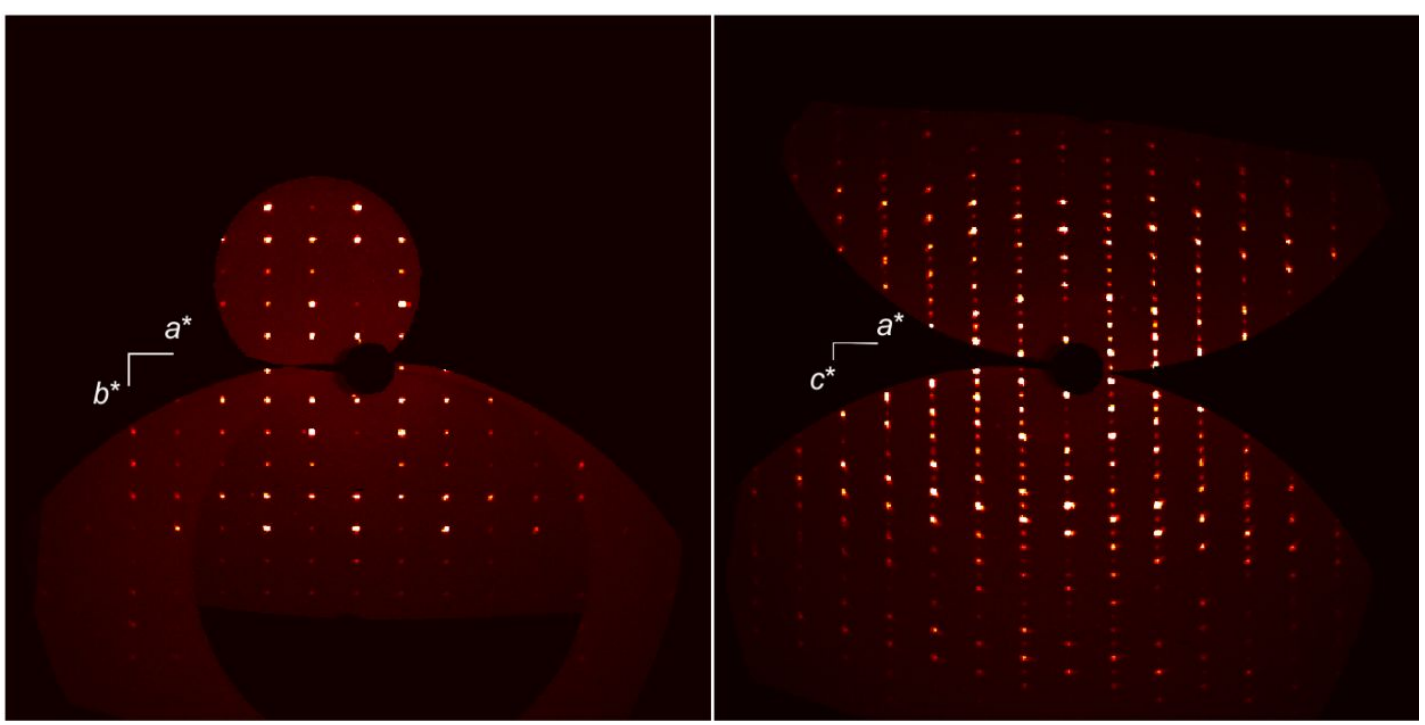

$h k 0$

hOl

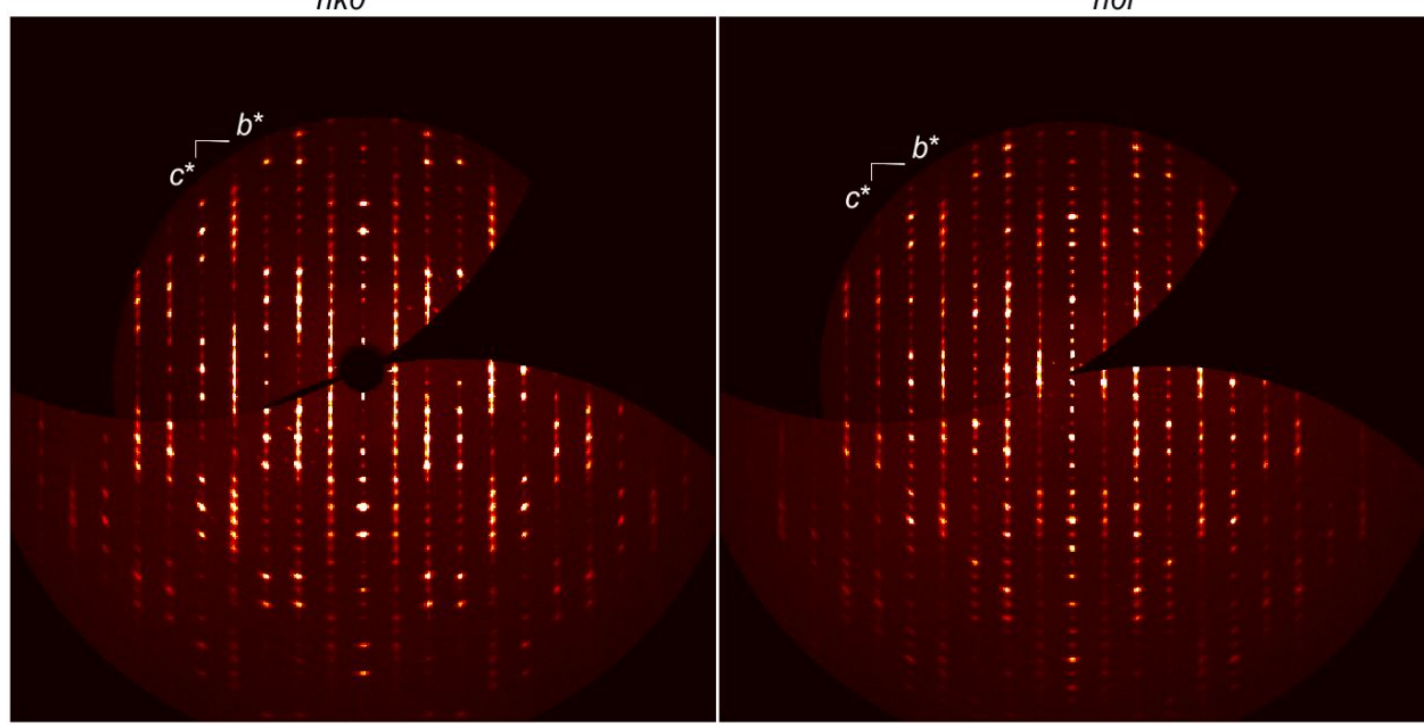

Okl

$1 k l$

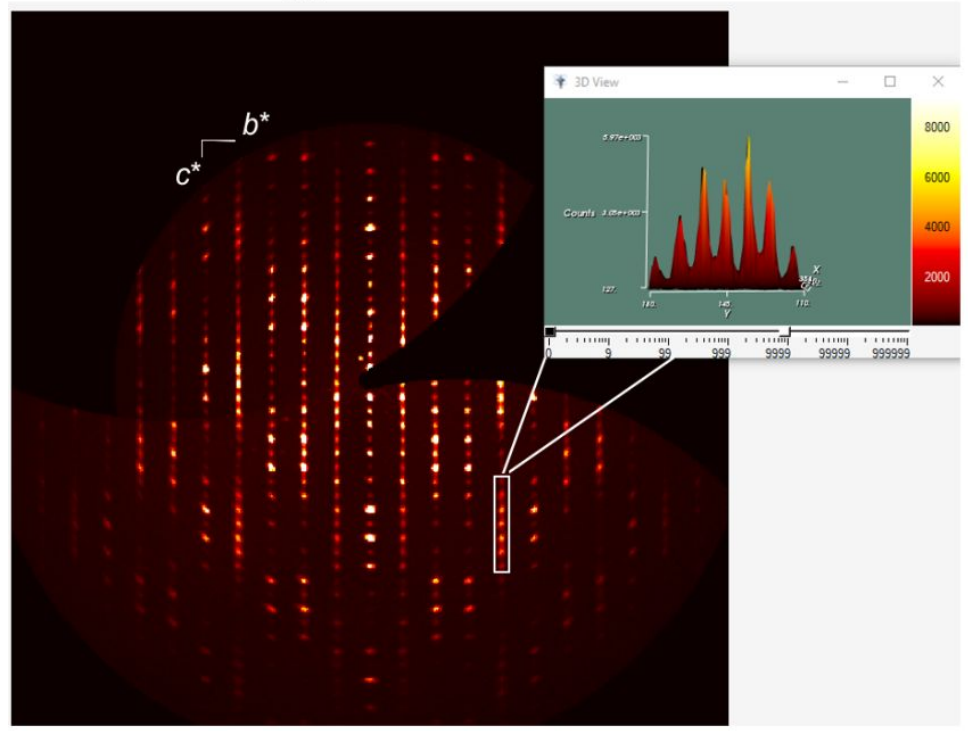

$2 k l$

Figure S1. Reciprocal space reconstructions for 2, the thickness of the layers of $0.10 \AA$. . 3D view of some reflections is in the inset to the image of $2 k$ l layer. 
Counts

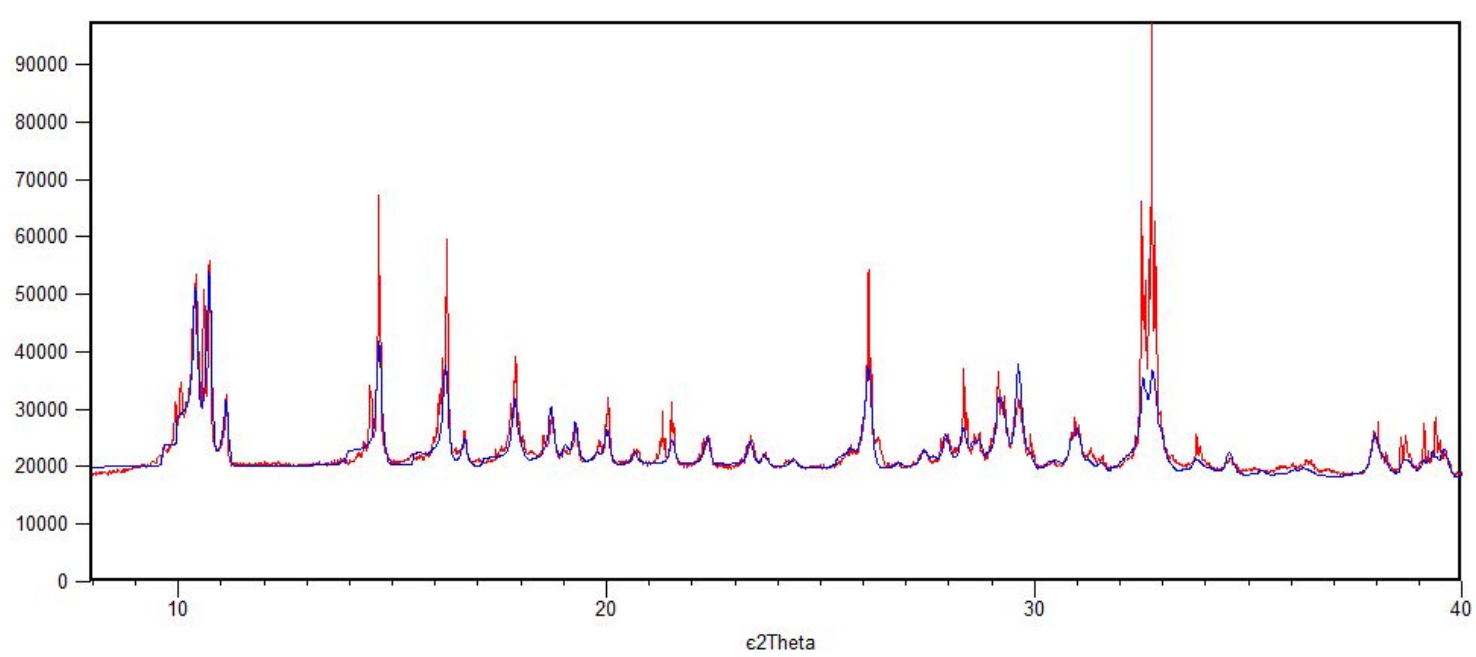

Figure S2. Calculated (blue) and experimental (red) PXRD patterns for 1

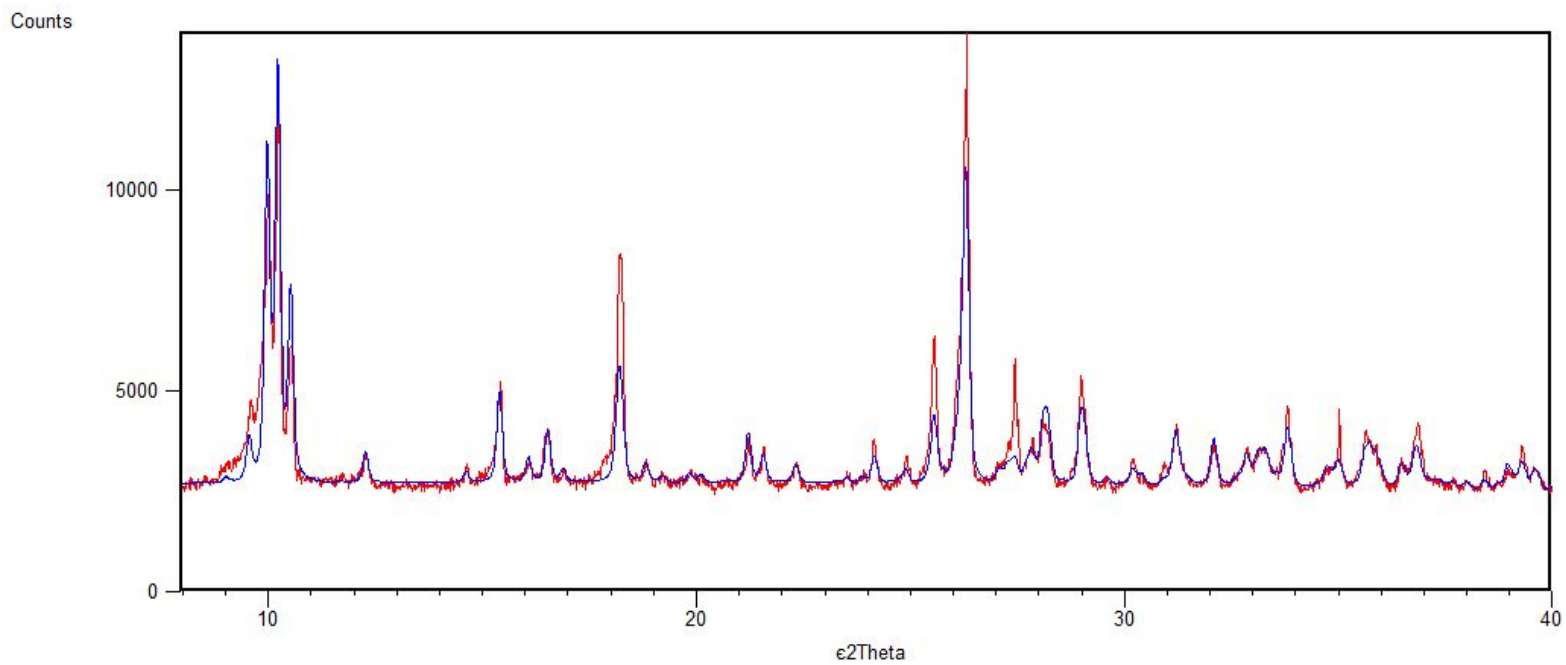

Figure S3. Calculated (blue) and experimental (red) PXRD patterns for 4 


\section{S-5}

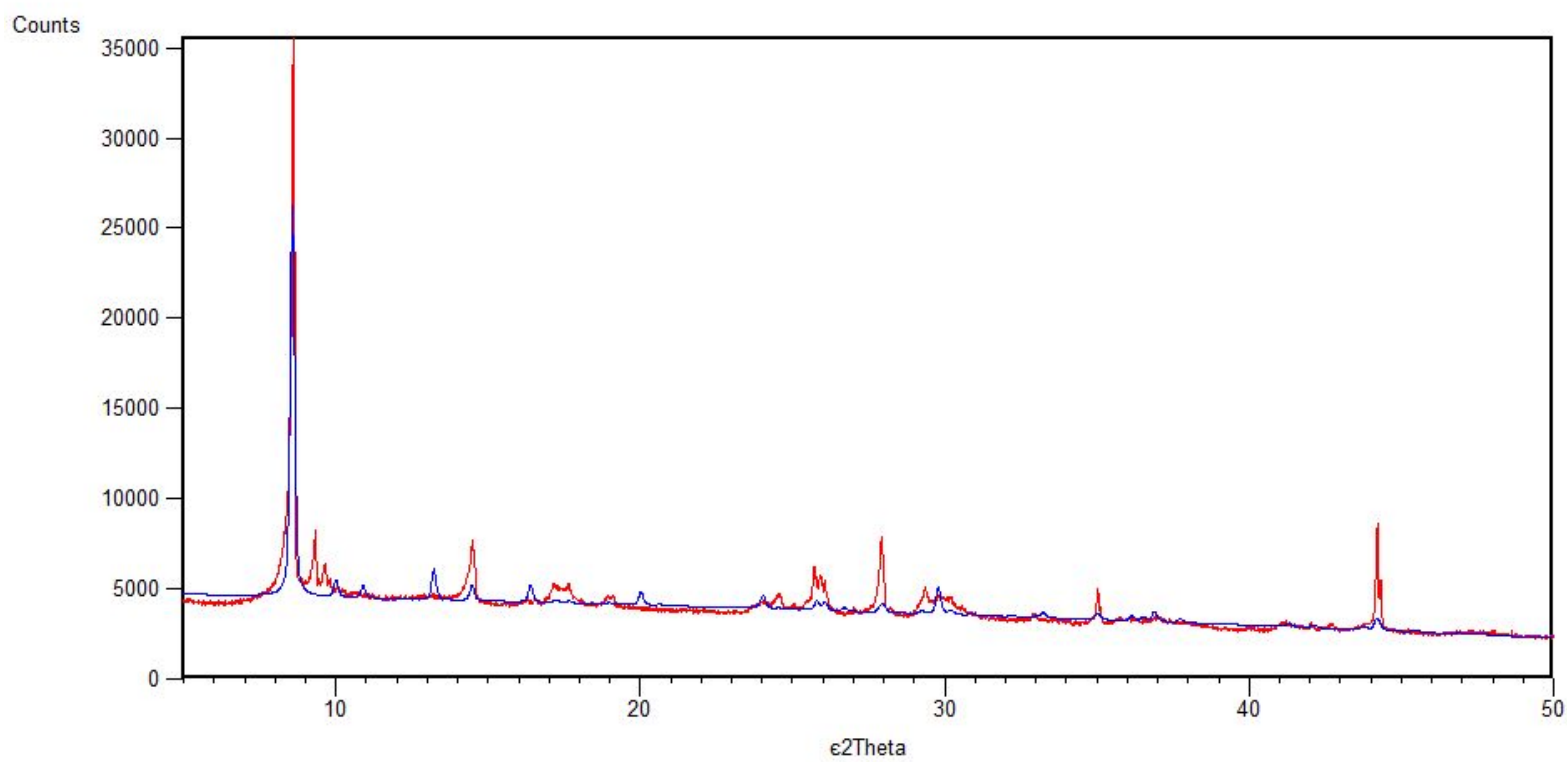

Figure S4. Calculated (blue) and experimental (red) PXRD patterns for $\mathbf{2}$

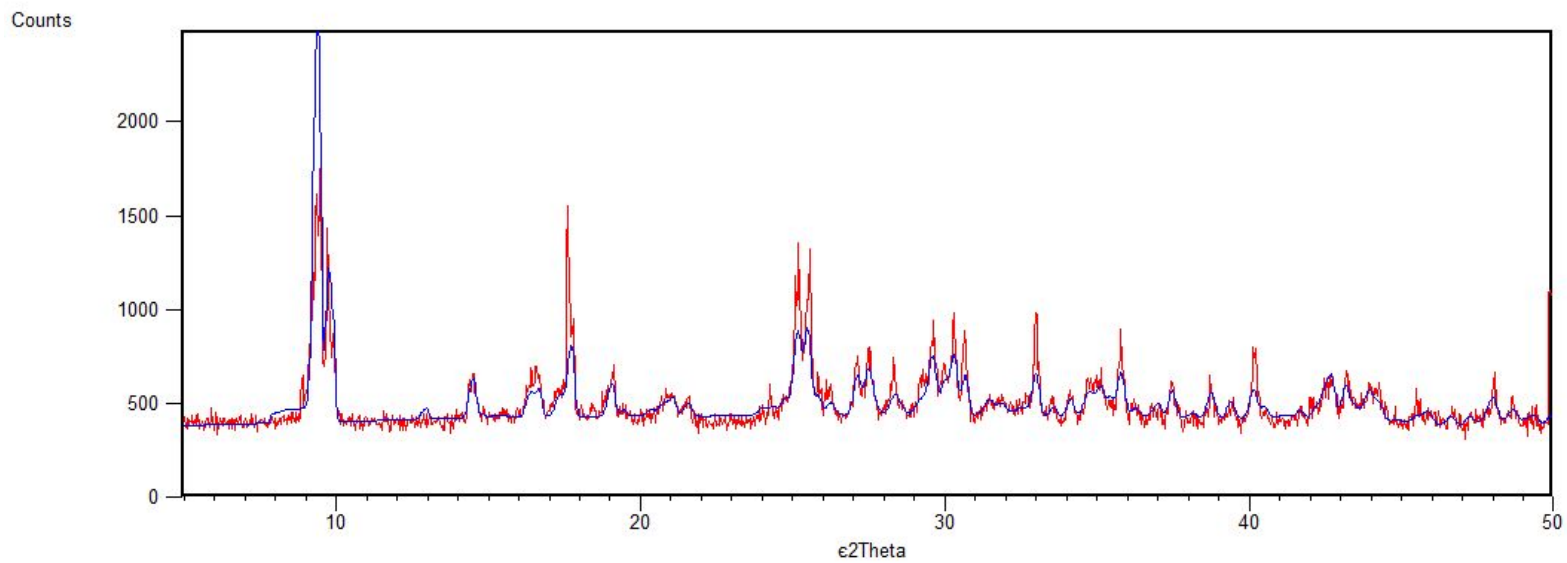

Figure S5. Calculated (blue) and experimental (red) PXRD patterns for $\mathbf{5}$

Diffuse reflectance spectra were measured on an setup which consists of a Kolibri-2 spectrometer (VMK Optoelektronica, Russia), fiber optic cable QR-400-7 (Ocean Optics, USA), and deuterium-tungsten lamp AvaLight-DHS (Avantes, Netherlands) (see V.R. Shayapov et al., New J. Chem. 2019, 43(9), 3927). The reference of $100 \%$ reflectance was $\mathrm{BaSO}_{4}$ powder. The spectra were recorded five times in the wavelength interval of 300-1000 nm and then averaged to reduce the random error. Kubelka-Munk function $F(R)$ was calculated using diffuse reflectance spectra: 


$$
F(R)=\frac{(1-R)^{2}}{2 R},(1)
$$

where $R$ is a diffuse reflectance coefficient. The Kubelka-Munk function represents as $k / s$ ratio where $k$ is absorption coefficient and $s$ is a scattering coefficient:

$$
F(R) \sim \frac{k}{s}(2)
$$

Energy band gap $E_{g}$ was determined by the Tauc method. The dependence of the $\sqrt{F(R) \cdot E}$ value (where $E=1240 / \lambda$ is photon energy) versus $E$ was plotted. The energy band gap was obtained by extrapolation of the linear portion of the dependence to the intersection with the energy axis.

\section{Computational details}

The single point calculations based on the experimental X-ray geometries of $\mathbf{1}, \mathbf{3}$, and $\mathbf{5}$ have been carried out at the DFT level of theory using the dispersion-corrected hybrid functional wB97XD [Phys. Chem. Chem. Phys. 2008, 10, 6615.] with the help of Gaussian-09 [M. J. Frisch, G. W. Trucks, H. B. Schlegel, G. E. Scuseria, M. A. Robb, J. R. Cheeseman, G. Scalmani, V. Barone, B. Mennucci, G. A. Petersson, H. Nakatsuji, M. Caricato, X. Li, H. P. Hratchian, A. F. Izmaylov, J. Bloino, G. Zheng, J. L. Sonnenberg, M. Hada, M. Ehara, K. Toyota, R. Fukuda, J. Hasegawa, M. Ishida, T. Nakajima, Y. Honda, O. Kitao, H. Nakai, T. Vreven, M. J. A.;, J. E. Peralta, F. Ogliaro, M. Bearpark, J. J. Heyd, E. Brothers, K. N. Kudin, V. N. Staroverov, T. Keith, R. Kobayashi, J. Normand, K. Raghavachari, A. Rendell, J. C. Burant, S. S. Iyengar, J. Tomasi, M. Cossi, N. Rega, J. M. Millam, M. Klene, J. E. Knox, J. B. Cross, V. Bakken, C. Adamo, J. Jaramillo, R. Gomperts, R. E. Stratmann, O. Yazyev, A. J. Austin, R. Cammi, C. Pomelli, J. W. Ochterski, R. L. Martin, K. Morokuma, V. G. Zakrzewski, G. A. Voth, P. Salvador, J. J. Dannenberg, S. Dapprich, A. D. Daniels, O. Farkas, J. B. Foresman, J. V. Ortiz, C. J.;, D. J. Fox, in Gaussian 09, Revision C.01, Gaussian, Inc., Wallingford, CT, 2010.] program package. The Douglas-Kroll-Hess $2^{\text {nd }}$ order scalar relativistic calculations requested relativistic core Hamiltonian were carried out using the DZP-DKH basis sets [Mol. Phys. 2010, 108, 1965. || J. Chem. Phys. 2009, 130, 064108. || Chem. Phys. Lett. 2013, 582, 158. II J. Mol. Struct. - Theochem 2010, 961, 107.] for all atoms. The topological analysis of the electron density distribution with the help of the atoms in molecules (QTAIM) method developed by Bader [Chem. Rev. 1991, 91, 893.] has been performed by using the Multiwfn program (version 3.7) [J. Comput. Chem. 2012, 33, 580.]. The Cartesian atomic coordinates for model supramolecular associates presented in Table S3. 


\section{S-7}
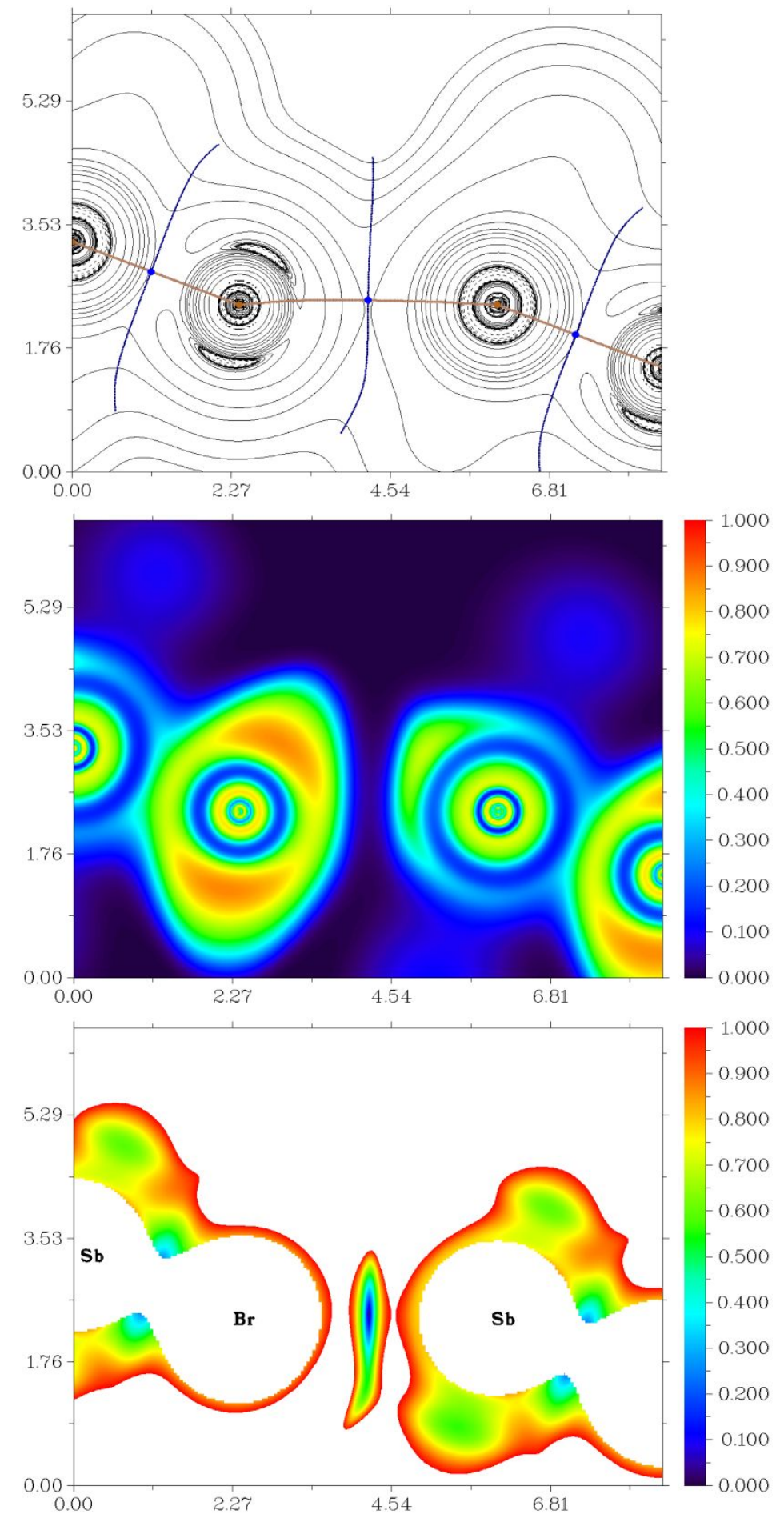

Figure S6. Contour line diagram of the Laplacian of electron density distribution $\nabla^{2} \rho(\mathbf{r})$, bond paths, and selected zero-flux surfaces (top), visualization of electron localization function (ELF, center) and reduced density gradient (RDG, bottom) analyses for intermolecular interactions $\mathrm{Sb} \cdots \mathrm{Br}$ in 1 . Bond critical points $(3,-1)$ are shown in blue, nuclear critical points $(3,-3)$ - in pale brown, bond paths are shown as pale brown lines, length units $-\AA$, and the color scale for the ELF and RDG maps are presented in a.u. 

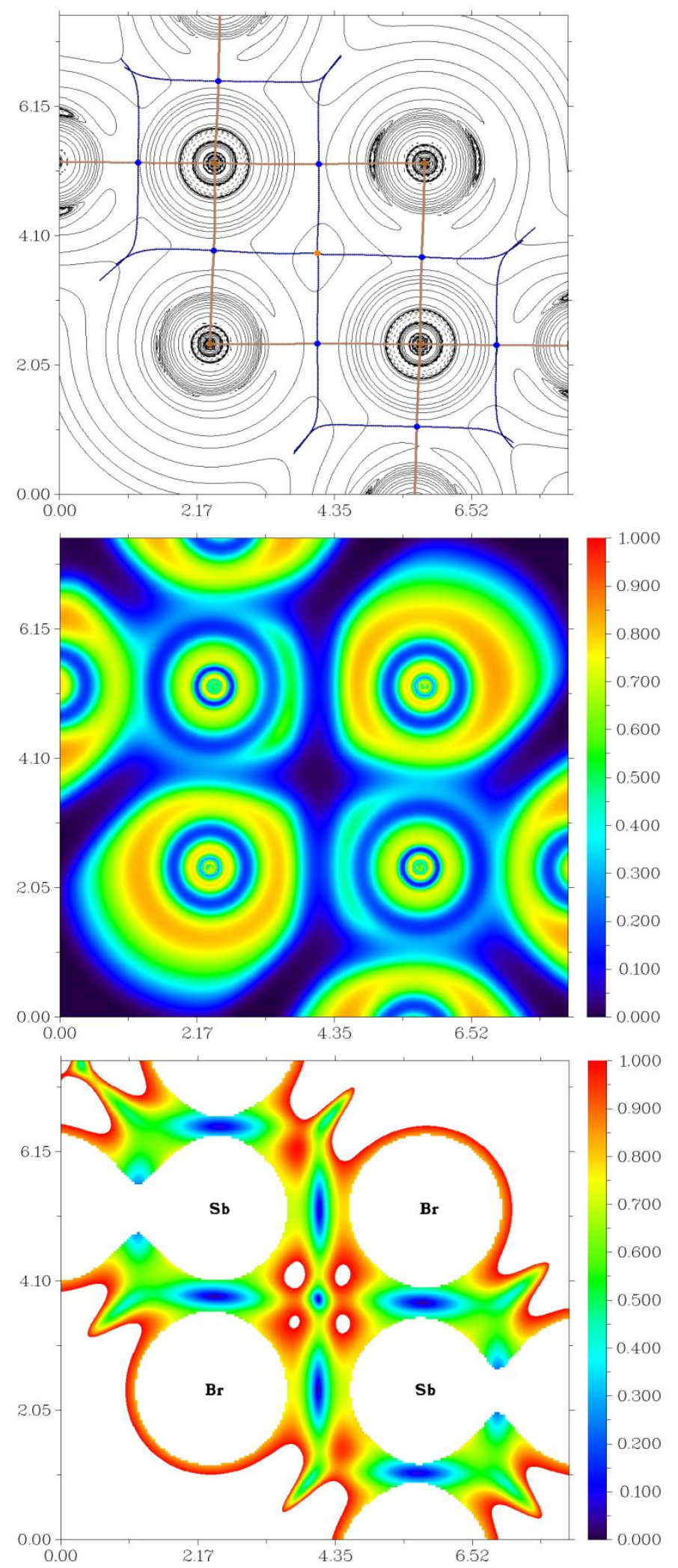

Figure S7. Contour line diagram of the Laplacian of electron density distribution $\nabla^{2} \rho(\mathbf{r})$, bond paths, and selected zero-flux surfaces (top), visualization of electron localization function (ELF, center) and reduced density gradient (RDG, bottom) analyses for intermolecular interactions $\mathrm{Sb} \cdots \mathrm{Br}$ in $\mathbf{3}$. Bond critical points $(3,-1)$ are shown in blue, nuclear critical points $(3,-3)$ - in pale brown, ring critical points $(3,+1)-$ in orange, bond paths are shown as pale brown lines, length units $-\AA$, and the color scale for the ELF and RDG maps are presented in a.u. 

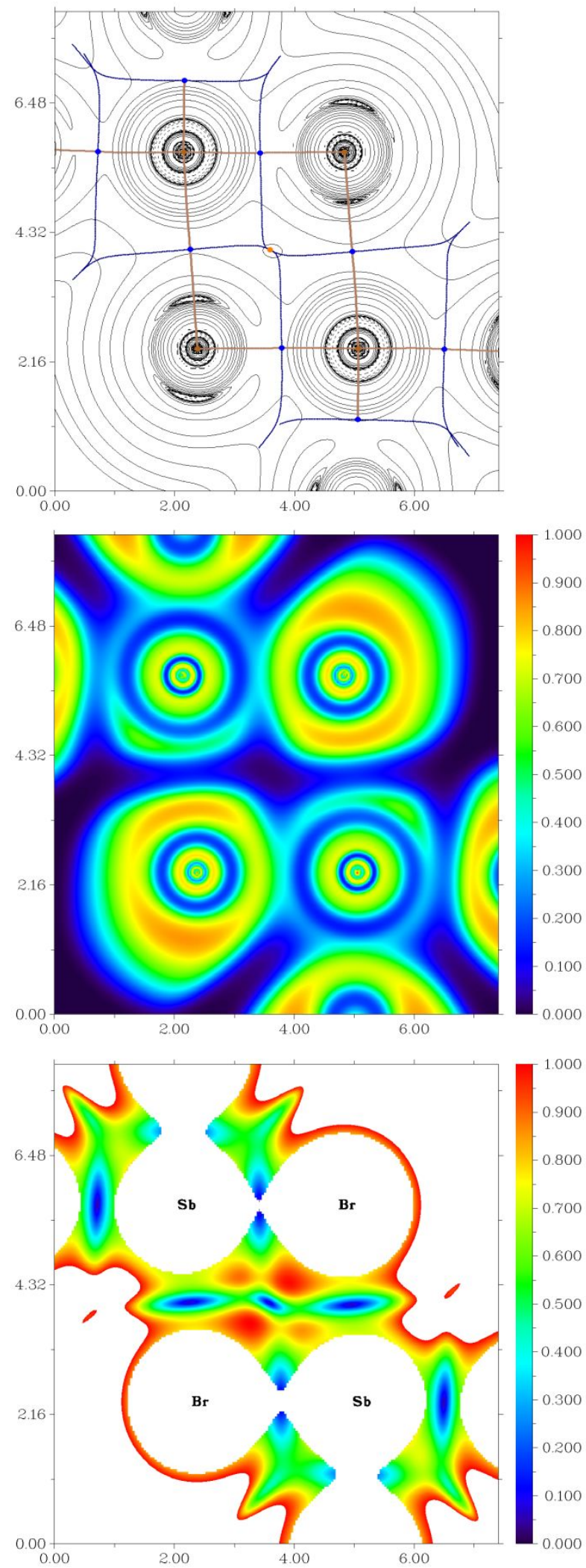

Figure S8. Contour line diagram of the Laplacian of electron density distribution $\nabla^{2} \rho(\mathbf{r})$, bond paths, and selected zero-flux surfaces (top), visualization of electron localization function (ELF, center) and reduced density gradient (RDG, bottom) analyses for intermolecular interactions $\mathrm{Sb} \cdots \mathrm{Br}$ in $\mathbf{5}$. Bond critical points $(3,-1)$ are shown in blue, nuclear critical points $(3,-3)$ - in pale brown, ring critical points $(3,+1)-$ in orange, bond paths are shown as pale brown lines, length units $-\AA$, and the color scale for the ELF and RDG maps are presented in a.u. 

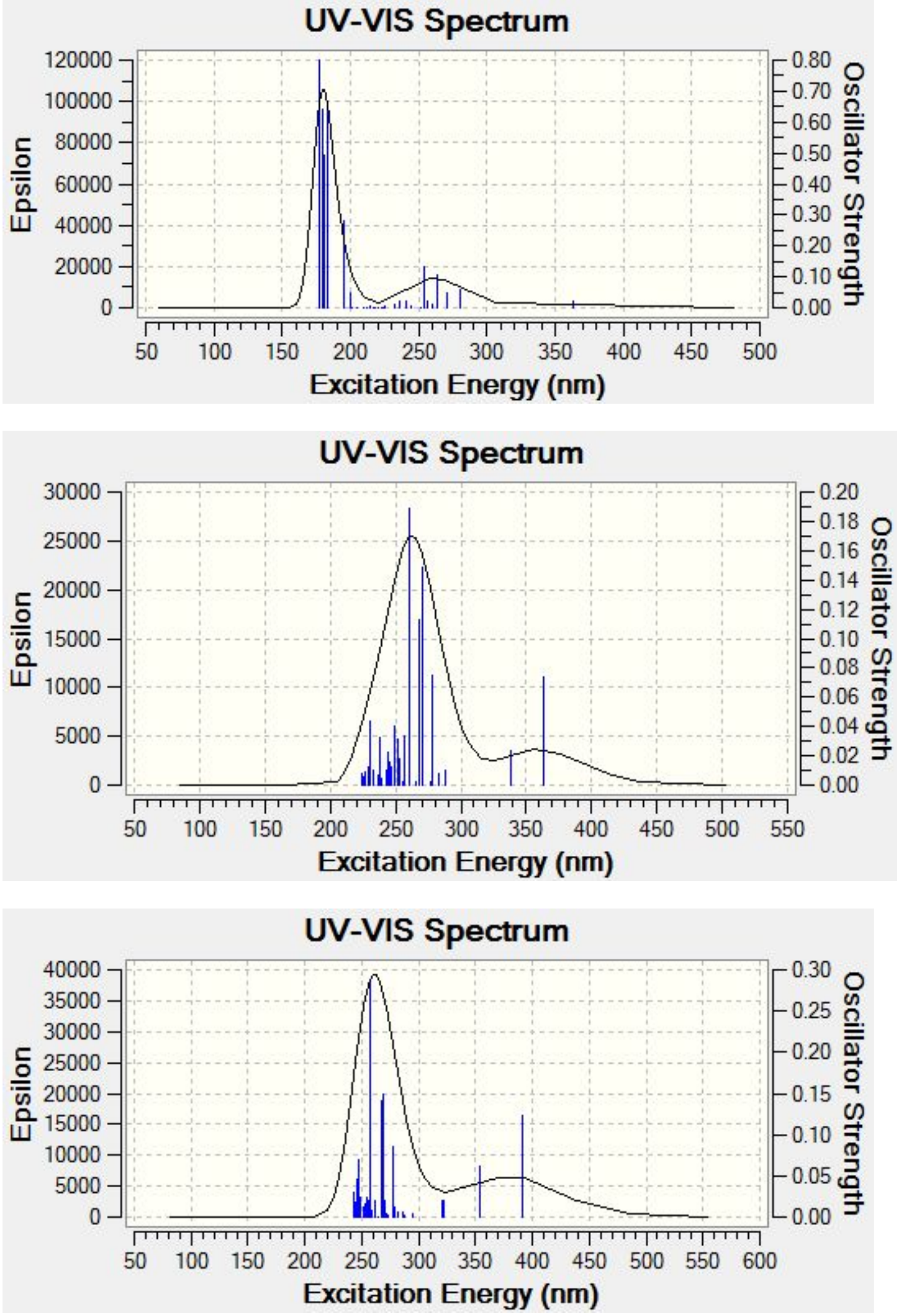

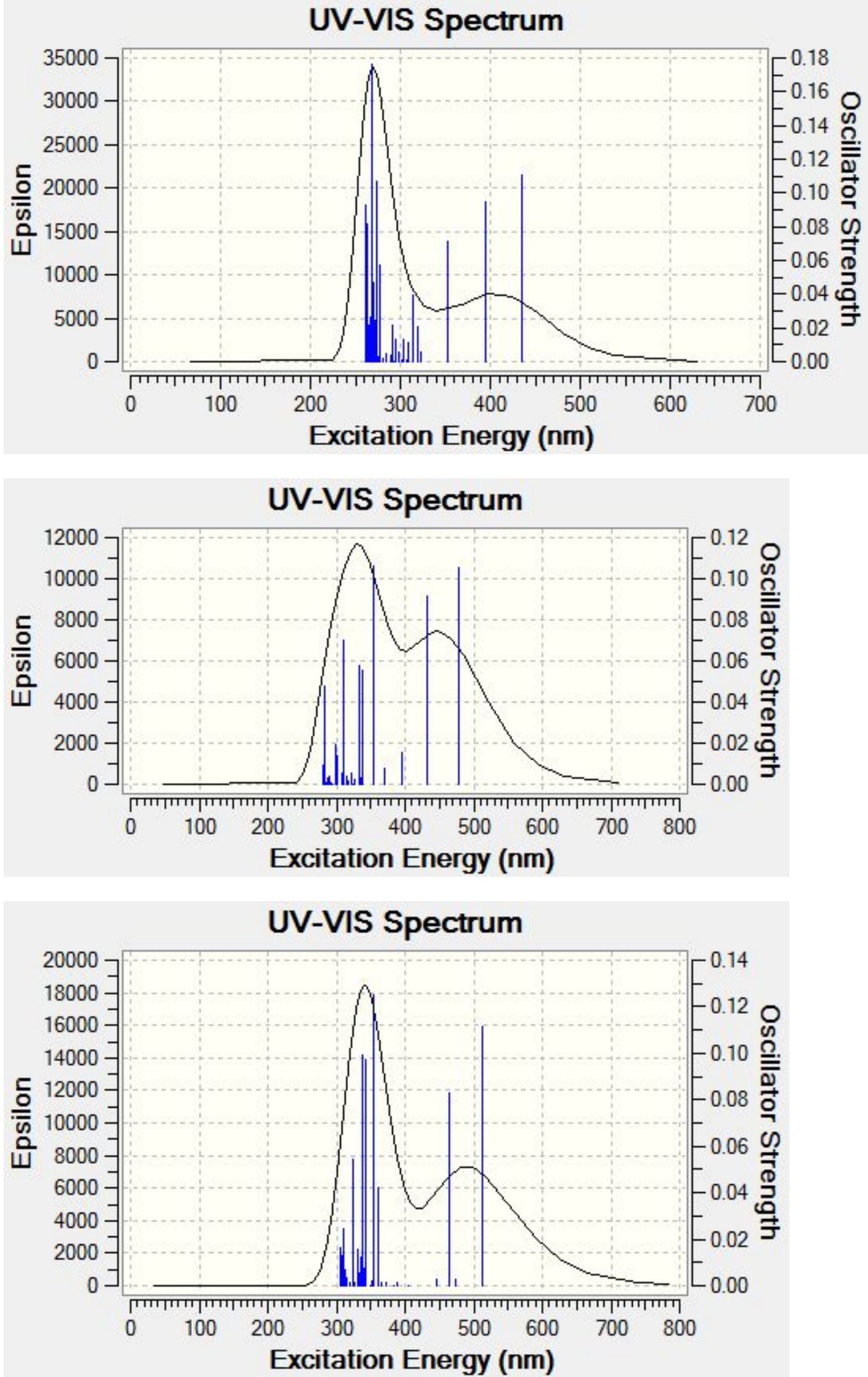

Figure S9. Theoretical UV-Vis absorption spectra for model mono-, di-, tri-, tetra-, penta-, and hexameric systems in case of $\mathbf{1}$ (from top to bottom, respectively). 

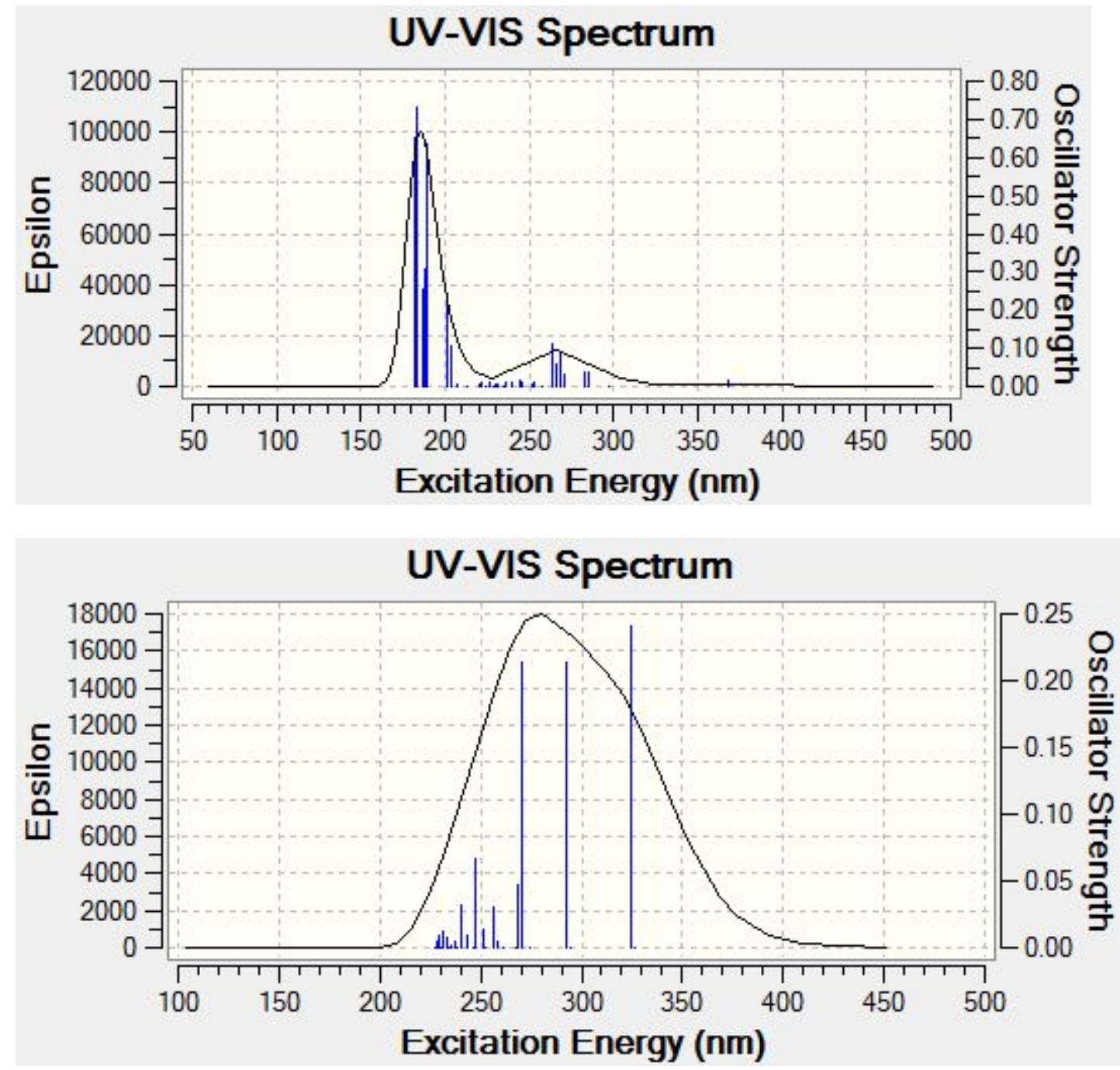

Figure S10. Theoretical UV-Vis absorption spectra for monomeric (top) and dimeric (bottom) systems in case of 3.

Table S3. Cartesian atomic coordinates for model supramolecular associates.

\begin{tabular}{|c|c|c|c|}
\hline Atom & $X$ & $Y$ & $Z$ \\
\hline 1_monomer & & & \\
\hline $\mathrm{Sb}$ & 1.119074 & 6.308693 & 12.647217 \\
\hline $\mathrm{Br}$ & 0.664157 & 4.850106 & 10.361041 \\
\hline $\mathrm{Br}$ & 1.602296 & 3.997682 & 14.043046 \\
\hline $\mathrm{Br}$ & 0.490502 & 8.699483 & 11.253418 \\
\hline $\mathrm{Br}$ & 1.554998 & 7.731337 & 14.957584 \\
\hline $\mathrm{Br}$ & -1.369126 & 6.124006 & 13.158113 \\
\hline 1_dimer & & & \\
\hline $\mathrm{Sb}$ & 1.119074 & 6.308693 & 12.647217 \\
\hline $\mathrm{Br}$ & 0.664157 & 4.850106 & 10.361041 \\
\hline $\mathrm{Br}$ & 1.602296 & 3.997682 & 14.043046 \\
\hline $\mathrm{Br}$ & 0.490502 & 8.699483 & 11.253418 \\
\hline
\end{tabular}




\begin{tabular}{|c|c|c|c|}
\hline $\mathrm{Br}$ & 1.554998 & 7.731337 & 14.957584 \\
\hline $\mathrm{Br}$ & -1.369126 & 6.124006 & 13.158113 \\
\hline $\mathrm{Sb}$ & 7.253374 & 6.308693 & 12.647217 \\
\hline $\mathrm{Br}$ & 6.798457 & 4.850106 & 10.361041 \\
\hline $\mathrm{Br}$ & 7.736596 & 3.997682 & 14.043046 \\
\hline $\mathrm{Br}$ & 6.624802 & 8.699483 & 11.253418 \\
\hline $\mathrm{Br}$ & 7.689298 & 7.731337 & 14.957584 \\
\hline $\mathrm{Br}$ & 4.765174 & 6.124006 & 13.158113 \\
\hline \multicolumn{4}{|c|}{ 1_trimer } \\
\hline $\mathrm{Sb}$ & 1.119074 & 6.308693 & 12.647217 \\
\hline $\mathrm{Br}$ & 0.664157 & 4.850106 & 10.361041 \\
\hline $\mathrm{Br}$ & 1.602296 & 3.997682 & 14.043046 \\
\hline $\mathrm{Br}$ & 0.490502 & 8.699483 & 11.253418 \\
\hline $\mathrm{Br}$ & 1.554998 & 7.731337 & 14.957584 \\
\hline $\mathrm{Br}$ & -1.369126 & 6.124006 & 13.158113 \\
\hline $\mathrm{Sb}$ & 7.253374 & 6.308693 & 12.647217 \\
\hline $\mathrm{Br}$ & 6.798457 & 4.850106 & 10.361041 \\
\hline $\mathrm{Br}$ & 7.736596 & 3.997682 & 14.043046 \\
\hline $\mathrm{Br}$ & 6.624802 & 8.699483 & 11.253418 \\
\hline $\mathrm{Br}$ & 7.689298 & 7.731337 & 14.957584 \\
\hline $\mathrm{Br}$ & 4.765174 & 6.124006 & 13.158113 \\
\hline $\mathrm{Sb}$ & -5.015226 & 6.308693 & 12.647217 \\
\hline $\mathrm{Br}$ & -5.470143 & 4.850106 & 10.361041 \\
\hline $\mathrm{Br}$ & -4.532004 & 3.997682 & 14.043046 \\
\hline $\mathrm{Br}$ & -5.643798 & 8.699483 & 11.253418 \\
\hline $\mathrm{Br}$ & -4.579302 & 7.731337 & 14.957584 \\
\hline $\mathrm{Br}$ & -7.503426 & 6.124006 & 13.158113 \\
\hline \multicolumn{4}{|c|}{ 1_tetramer } \\
\hline $\mathrm{Sb}$ & 1.119074 & 6.308693 & 12.647217 \\
\hline $\mathrm{Br}$ & 0.664157 & 4.850106 & 10.361041 \\
\hline $\mathrm{Br}$ & 1.602296 & 3.997682 & 14.043046 \\
\hline $\mathrm{Br}$ & 0.490502 & 8.699483 & 11.253418 \\
\hline $\mathrm{Br}$ & 1.554998 & 7.731337 & 14.957584 \\
\hline $\mathrm{Br}$ & -1.369126 & 6.124006 & 13.158113 \\
\hline $\mathrm{Sb}$ & -5.015226 & 6.308693 & 12.647217 \\
\hline $\mathrm{Br}$ & -5.470143 & 4.850106 & 10.361041 \\
\hline $\mathrm{Br}$ & -4.532004 & 3.997682 & 14.043046 \\
\hline $\mathrm{Br}$ & -5.643798 & 8.699483 & 11.253418 \\
\hline $\mathrm{Br}$ & -4.579302 & 7.731337 & 14.957584 \\
\hline $\mathrm{Br}$ & -7.503426 & 6.124006 & 13.158113 \\
\hline $\mathrm{Sb}$ & -11.149526 & 6.308693 & 12.647217 \\
\hline $\mathrm{Br}$ & -11.604443 & 4.850106 & 10.361041 \\
\hline $\mathrm{Br}$ & -10.666304 & 3.997682 & 14.043046 \\
\hline $\mathrm{Br}$ & -11.778098 & 8.699483 & 11.253418 \\
\hline $\mathrm{Br}$ & -10.713602 & 7.731337 & 14.957584 \\
\hline $\mathrm{Br}$ & -13.637726 & 6.124006 & 13.158113 \\
\hline $\mathrm{Sb}$ & -17.283826 & 6.308693 & 12.647217 \\
\hline $\mathrm{Br}$ & -17.738743 & 4.850106 & 10.361041 \\
\hline $\mathrm{Br}$ & -16.800604 & 3.997682 & 14.043046 \\
\hline $\mathrm{Br}$ & -17.912398 & 8.699483 & 11.253418 \\
\hline $\mathrm{Br}$ & -16.847902 & 7.731337 & 14.957584 \\
\hline
\end{tabular}




\begin{tabular}{|c|c|c|c|}
\hline $\mathrm{Br}$ & -19.772026 & 6.124006 & 13.158113 \\
\hline \multicolumn{4}{|c|}{ 1_pentamer } \\
\hline $\mathrm{Sb}$ & 1.119074 & 6.308693 & 12.647217 \\
\hline $\mathrm{Br}$ & 0.664157 & 4.850106 & 10.361041 \\
\hline $\mathrm{Br}$ & 1.602296 & 3.997682 & 14.043046 \\
\hline $\mathrm{Br}$ & 0.490502 & 8.699483 & 11.253418 \\
\hline $\mathrm{Br}$ & 1.554998 & 7.731337 & 14.957584 \\
\hline $\mathrm{Br}$ & -1.369126 & 6.124006 & 13.158113 \\
\hline $\mathrm{Sb}$ & -5.015226 & 6.308693 & 12.647217 \\
\hline $\mathrm{Br}$ & -5.470143 & 4.850106 & 10.361041 \\
\hline $\mathrm{Br}$ & -4.532004 & 3.997682 & 14.043046 \\
\hline $\mathrm{Br}$ & -5.643798 & 8.699483 & 11.253418 \\
\hline $\mathrm{Br}$ & -4.579302 & 7.731337 & 14.957584 \\
\hline $\mathrm{Br}$ & -7.503426 & 6.124006 & 13.158113 \\
\hline $\mathrm{Sb}$ & -11.149526 & 6.308693 & 12.647217 \\
\hline $\mathrm{Br}$ & -11.604443 & 4.850106 & 10.361041 \\
\hline $\mathrm{Br}$ & -10.666304 & 3.997682 & 14.043046 \\
\hline $\mathrm{Br}$ & -11.778098 & 8.699483 & 11.253418 \\
\hline $\mathrm{Br}$ & -10.713602 & 7.731337 & 14.957584 \\
\hline $\mathrm{Br}$ & -13.637726 & 6.124006 & 13.158113 \\
\hline $\mathrm{Sb}$ & -17.283826 & 6.308693 & 12.647217 \\
\hline $\mathrm{Br}$ & -17.738743 & 4.850106 & 10.361041 \\
\hline $\mathrm{Br}$ & -16.800604 & 3.997682 & 14.043046 \\
\hline $\mathrm{Br}$ & -17.912398 & 8.699483 & 11.253418 \\
\hline $\mathrm{Br}$ & -16.847902 & 7.731337 & 14.957584 \\
\hline $\mathrm{Br}$ & -19.772026 & 6.124006 & 13.158113 \\
\hline $\mathrm{Sb}$ & -23.418126 & 6.308693 & 12.647217 \\
\hline $\mathrm{Br}$ & -23.873043 & 4.850106 & 10.361041 \\
\hline $\mathrm{Br}$ & -22.934904 & 3.997682 & 14.043046 \\
\hline $\mathrm{Br}$ & -24.046698 & 8.699483 & 11.253418 \\
\hline $\mathrm{Br}$ & -22.982202 & 7.731337 & 14.957584 \\
\hline $\mathrm{Br}$ & -25.906326 & 6.124006 & 13.158113 \\
\hline \multicolumn{4}{|c|}{ 1_hexamer } \\
\hline $\mathrm{Sb}$ & 1.119074 & 6.308693 & 12.647217 \\
\hline $\mathrm{Br}$ & 0.664157 & 4.850106 & 10.361041 \\
\hline $\mathrm{Br}$ & 1.602296 & 3.997682 & 14.043046 \\
\hline $\mathrm{Br}$ & 0.490502 & 8.699483 & 11.253418 \\
\hline $\mathrm{Br}$ & 1.554998 & 7.731337 & 14.957584 \\
\hline $\mathrm{Br}$ & -1.369126 & 6.124006 & 13.158113 \\
\hline $\mathrm{Sb}$ & -5.015226 & 6.308693 & 12.647217 \\
\hline $\mathrm{Br}$ & -5.470143 & 4.850106 & 10.361041 \\
\hline $\mathrm{Br}$ & -4.532004 & 3.997682 & 14.043046 \\
\hline $\mathrm{Br}$ & -5.643798 & 8.699483 & 11.253418 \\
\hline $\mathrm{Br}$ & -4.579302 & 7.731337 & 14.957584 \\
\hline $\mathrm{Br}$ & -7.503426 & 6.124006 & 13.158113 \\
\hline $\mathrm{Sb}$ & -11.149526 & 6.308693 & 12.647217 \\
\hline $\mathrm{Br}$ & -11.604443 & 4.850106 & 10.361041 \\
\hline $\mathrm{Br}$ & -10.666304 & 3.997682 & 14.043046 \\
\hline $\mathrm{Br}$ & -11.778098 & 8.699483 & 11.253418 \\
\hline $\mathrm{Br}$ & -10.713602 & 7.731337 & 14.957584 \\
\hline $\mathrm{Br}$ & -13.637726 & 6.124006 & 13.158113 \\
\hline
\end{tabular}




\begin{tabular}{|c|c|c|c|}
\hline $\mathrm{Sb}$ & -17.283826 & 6.308693 & 12.647217 \\
\hline $\mathrm{Br}$ & -17.738743 & 4.850106 & 10.361041 \\
\hline $\mathrm{Br}$ & -16.800604 & 3.997682 & 14.043046 \\
\hline $\mathrm{Br}$ & -17.912398 & 8.699483 & 11.253418 \\
\hline $\mathrm{Br}$ & -16.847902 & 7.731337 & 14.957584 \\
\hline $\mathrm{Br}$ & -19.772026 & 6.124006 & 13.158113 \\
\hline $\mathrm{Sb}$ & 7.253374 & 6.308693 & 12.647217 \\
\hline $\mathrm{Br}$ & 6.798457 & 4.850106 & 10.361041 \\
\hline $\mathrm{Br}$ & 7.736596 & 3.997682 & 14.043046 \\
\hline $\mathrm{Br}$ & 6.624802 & 8.699483 & 11.253418 \\
\hline $\mathrm{Br}$ & 7.689298 & 7.731337 & 14.957584 \\
\hline $\mathrm{Br}$ & 4.765174 & 6.124006 & 13.158113 \\
\hline $\mathrm{Sb}$ & 13.387674 & 6.308693 & 12.647217 \\
\hline $\mathrm{Br}$ & 12.932757 & 4.850106 & 10.361041 \\
\hline $\mathrm{Br}$ & 13.870896 & 3.997682 & 14.043046 \\
\hline $\mathrm{Br}$ & 12.759102 & 8.699483 & 11.253418 \\
\hline $\mathrm{Br}$ & 13.823598 & 7.731337 & 14.957584 \\
\hline $\mathrm{Br}$ & 10.899474 & 6.124006 & 13.158113 \\
\hline \multicolumn{4}{|c|}{ 3_monomer } \\
\hline $\mathrm{Br}$ & 7.562629 & 2.403616 & 0.321306 \\
\hline $\mathrm{Br}$ & 6.414646 & -1.415344 & 1.050303 \\
\hline $\mathrm{Br}$ & 3.567261 & -0.025001 & 3.353009 \\
\hline $\mathrm{Br}$ & 7.150041 & 1.219746 & 3.720301 \\
\hline $\mathrm{Br}$ & 4.523123 & 3.686847 & 2.432585 \\
\hline $\mathrm{Sb}$ & 5.538693 & 1.237191 & 1.718451 \\
\hline \multicolumn{4}{|c|}{ 3_dimer } \\
\hline $\mathrm{Br}$ & 7.562629 & 2.403616 & 0.321306 \\
\hline $\mathrm{Br}$ & 6.414646 & -1.415344 & 1.050303 \\
\hline $\mathrm{Br}$ & 3.567261 & -0.025001 & 3.353009 \\
\hline $\mathrm{Br}$ & 7.150041 & 1.219746 & 3.720301 \\
\hline $\mathrm{Br}$ & 4.523123 & 3.686847 & 2.432585 \\
\hline $\mathrm{Sb}$ & 5.538693 & 1.237191 & 1.718451 \\
\hline $\mathrm{Br}$ & 2.543371 & -2.403616 & -0.321306 \\
\hline $\mathrm{Br}$ & 3.691354 & 1.415344 & -1.050303 \\
\hline $\mathrm{Br}$ & 6.538739 & 0.025001 & -3.353009 \\
\hline $\mathrm{Br}$ & 2.955959 & -1.219746 & -3.720301 \\
\hline $\mathrm{Br}$ & 5.582877 & -3.686847 & -2.432585 \\
\hline $\mathrm{Sb}$ & 4.567307 & -1.237191 & -1.718451 \\
\hline \multicolumn{4}{|c|}{ 1_QTAIM } \\
\hline $\mathrm{Sb}$ & 1.119074 & 6.308693 & 12.647217 \\
\hline $\mathrm{Br}$ & 0.664157 & 4.850106 & 10.361041 \\
\hline $\mathrm{Br}$ & 1.602296 & 3.997682 & 14.043046 \\
\hline $\mathrm{Br}$ & 0.490502 & 8.699483 & 11.253418 \\
\hline $\mathrm{Br}$ & 1.554998 & 7.731337 & 14.957584 \\
\hline $\mathrm{Br}$ & -1.369126 & 6.124006 & 13.158113 \\
\hline $\mathrm{Sb}$ & 7.253374 & 6.308693 & 12.647217 \\
\hline $\mathrm{Br}$ & 6.798457 & 4.850106 & 10.361041 \\
\hline $\mathrm{Br}$ & 7.736596 & 3.997682 & 14.043046 \\
\hline $\mathrm{Br}$ & 6.624802 & 8.699483 & 11.253418 \\
\hline $\mathrm{Br}$ & 7.689298 & 7.731337 & 14.957584 \\
\hline $\mathrm{Br}$ & 4.765174 & 6.124006 & 13.158113 \\
\hline
\end{tabular}




\begin{tabular}{|c|c|c|c|}
\hline $\mathrm{Sb}$ & -5.015226 & 6.308693 & 12.647217 \\
\hline $\mathrm{Br}$ & -5.470143 & 4.850106 & 10.361041 \\
\hline $\mathrm{Br}$ & -4.532004 & 3.997682 & 14.043046 \\
\hline $\mathrm{Br}$ & -5.643798 & 8.699483 & 11.253418 \\
\hline $\mathrm{Br}$ & -4.579302 & 7.731337 & 14.957584 \\
\hline $\mathrm{Br}$ & -7.503426 & 6.124006 & 13.158113 \\
\hline \multicolumn{4}{|c|}{ 3_QTAIM } \\
\hline $\mathrm{Br}$ & 7.562629 & 2.403616 & 0.321306 \\
\hline $\mathrm{Br}$ & 6.414646 & -1.415344 & 1.050303 \\
\hline $\mathrm{Br}$ & 3.567261 & -0.025001 & 3.353009 \\
\hline $\mathrm{Br}$ & 7.150041 & 1.219746 & 3.720301 \\
\hline $\mathrm{Br}$ & 4.523123 & 3.686847 & 2.432585 \\
\hline $\mathrm{Sb}$ & 5.538693 & 1.237191 & 1.718451 \\
\hline $\mathrm{Br}$ & 5.677358 & 2.338441 & 9.654028 \\
\hline $\mathrm{Br}$ & 6.825341 & 6.157401 & 8.925031 \\
\hline $\mathrm{Br}$ & 9.672726 & 4.767059 & 6.622325 \\
\hline $\mathrm{Br}$ & 6.089947 & 3.522311 & 6.255033 \\
\hline $\mathrm{Br}$ & 8.716864 & 1.055210 & 7.542749 \\
\hline $\mathrm{Sb}$ & 7.701294 & 3.504866 & 8.256883 \\
\hline $\mathrm{Br}$ & 2.543371 & -2.403616 & -0.321306 \\
\hline $\mathrm{Br}$ & 3.691354 & 1.415344 & -1.050303 \\
\hline $\mathrm{Br}$ & 6.538739 & 0.025001 & -3.353009 \\
\hline $\mathrm{Br}$ & 2.955959 & -1.219746 & -3.720301 \\
\hline $\mathrm{Br}$ & 5.582877 & -3.686847 & -2.432585 \\
\hline $\mathrm{Sb}$ & 4.567307 & -1.237191 & -1.718451 \\
\hline \multicolumn{4}{|c|}{ 5_QTAIM } \\
\hline $\mathrm{Br}$ & -1.532936 & 1.073229 & 8.531228 \\
\hline $\mathrm{Br}$ & -3.578836 & 4.299044 & 8.000226 \\
\hline $\mathrm{Br}$ & -3.987524 & -0.322107 & 11.193392 \\
\hline $\mathrm{Br}$ & -2.549589 & 3.047905 & 11.531713 \\
\hline $\mathrm{Sb}$ & -3.803680 & 1.864705 & 9.647613 \\
\hline $\mathrm{Br}$ & -8.343452 & 6.224171 & 10.285306 \\
\hline $\mathrm{Br}$ & -6.297552 & 2.998356 & 10.816308 \\
\hline $\mathrm{Br}$ & -5.888864 & 7.619507 & 7.623143 \\
\hline $\mathrm{Br}$ & -7.326799 & 4.249495 & 7.284821 \\
\hline $\mathrm{Sb}$ & -6.072708 & 5.432695 & 9.168921 \\
\hline $\mathrm{Br}$ & -1.532936 & -6.224171 & 8.531228 \\
\hline $\mathrm{Br}$ & -3.578836 & -2.998356 & 8.000226 \\
\hline $\mathrm{Br}$ & -3.987524 & -7.619507 & 11.193392 \\
\hline $\mathrm{Br}$ & -2.549589 & -4.249495 & 11.531713 \\
\hline $\mathrm{Sb}$ & -3.803680 & -5.432695 & 9.647613 \\
\hline $\mathrm{Br}$ & -8.343452 & -1.073229 & 10.285306 \\
\hline $\mathrm{Br}$ & -6.297552 & -4.299044 & 10.816308 \\
\hline $\mathrm{Br}$ & -5.888864 & 0.322107 & 7.623143 \\
\hline $\mathrm{Br}$ & -7.326799 & -3.047905 & 7.284821 \\
\hline $\mathrm{Sb}$ & -6.072708 & -1.864705 & 9.168921 \\
\hline $\mathrm{Br}$ & -1.532936 & 8.370629 & 8.531228 \\
\hline $\mathrm{Br}$ & -3.578836 & 11.596444 & 8.000226 \\
\hline $\mathrm{Br}$ & -3.987524 & 6.975293 & 11.193392 \\
\hline $\mathrm{Br}$ & -2.549589 & 10.345305 & 11.531713 \\
\hline $\mathrm{Sb}$ & -3.803680 & 9.162105 & 9.647613 \\
\hline
\end{tabular}




\section{S-17}

\begin{tabular}{|c|c|c|c|}
\hline $\mathrm{Br}$ & -8.343452 & 13.521571 & 10.285306 \\
\hline $\mathrm{Br}$ & -6.297552 & 10.295756 & 10.816308 \\
\hline $\mathrm{Br}$ & -5.888864 & 14.916907 & 7.623143 \\
\hline $\mathrm{Br}$ & -7.326799 & 11.546895 & 7.284821 \\
\hline $\mathrm{Sb}$ & -6.072708 & 12.730095 & 9.168921 \\
\hline
\end{tabular}

Table S4. Element analysis data for 1-5

\begin{tabular}{|c|c|c|}
\hline Compound & Calculated $(\mathrm{C}, \mathrm{H}, \mathrm{N}, \%)$ & Found $(\mathrm{C}, \mathrm{H}, \mathrm{N}, \%)$ \\
\hline $\mathbf{3 / 4}$ & $20.52,2.01,3.99$ & $20.60,2.05,4.02$ \\
\hline $\mathbf{5}$ & $17.21,1,99,2.51$ & $17.24 ; 2.03 ; 2.55$ \\
\hline
\end{tabular}

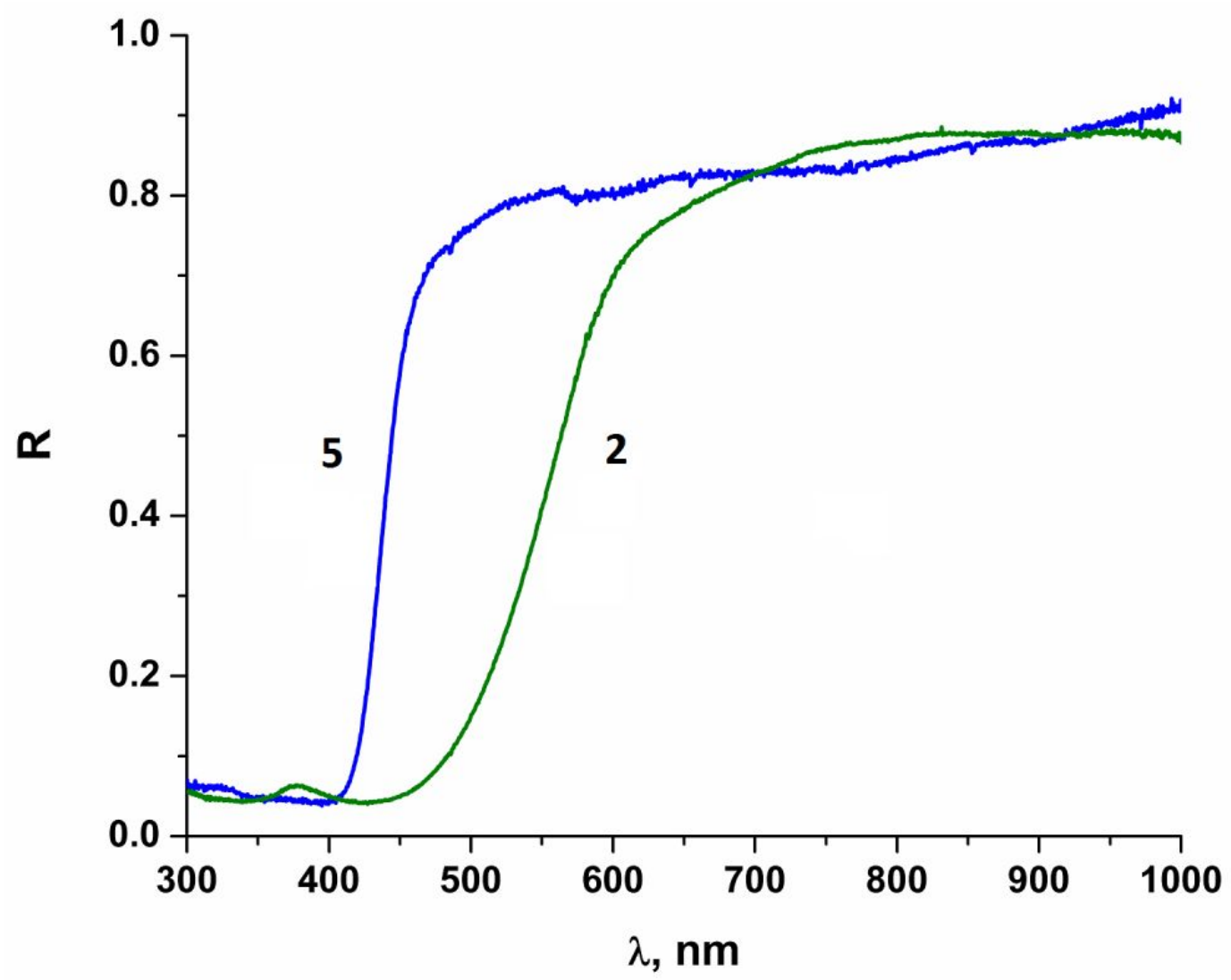

Figure S11. Diffuse reflectance spectra of $\mathbf{2}$ (blue) and $\mathbf{5}$ (green) 


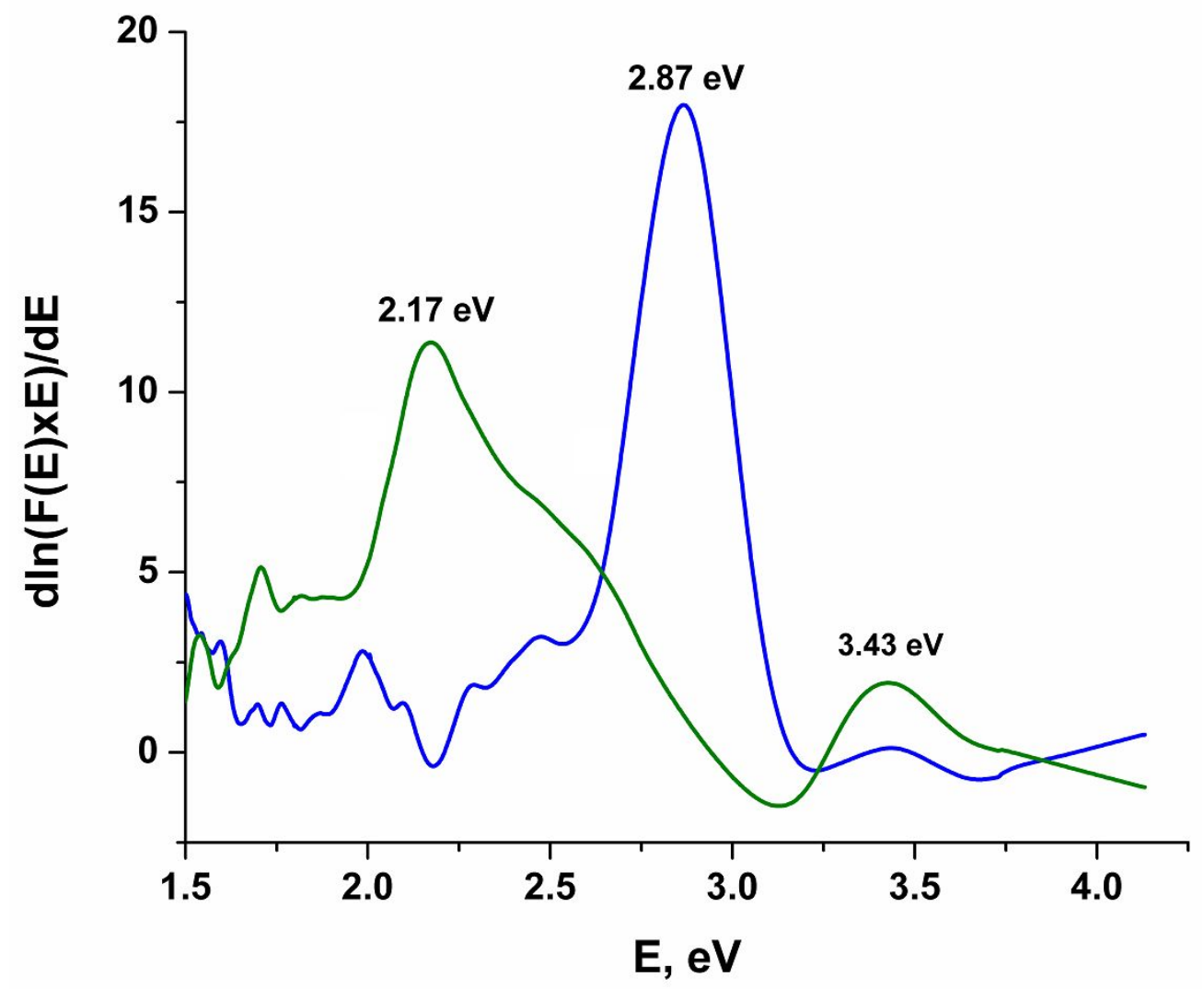

Figure S12. Diffuse reflectance spectra of $\mathbf{2}$ (green) and $\mathbf{5}$ (blue)

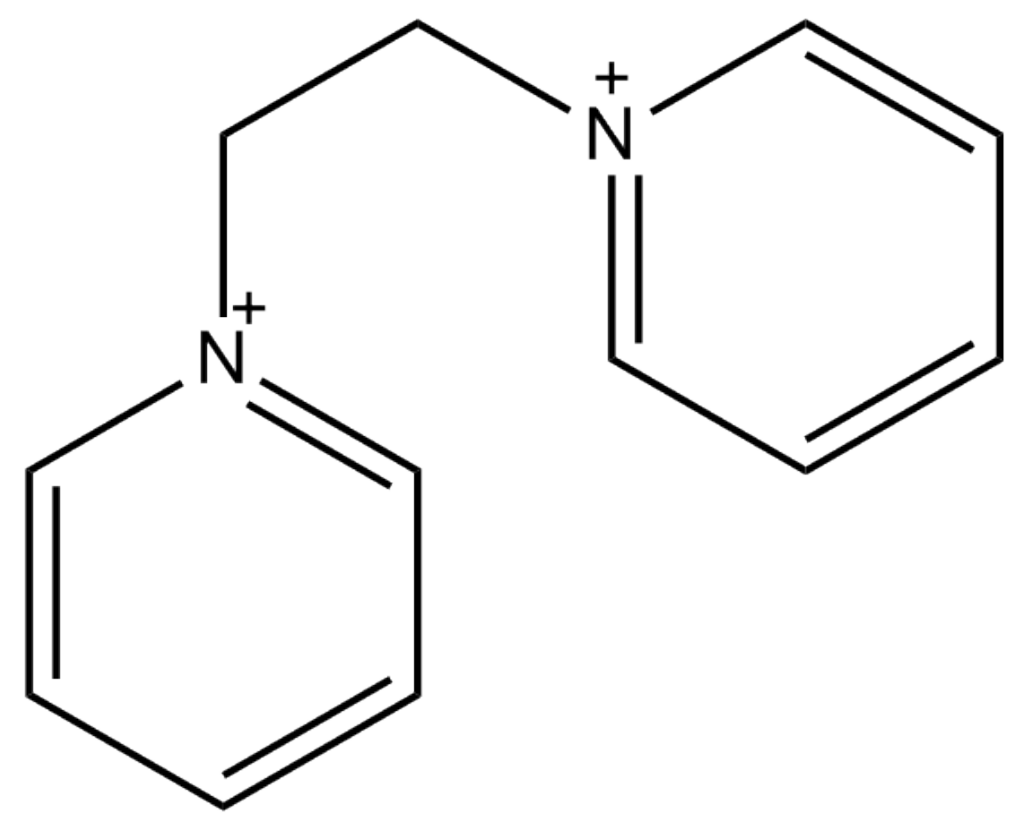

Figure S13. Structure of $\mathrm{PyC}_{2}{ }^{2+}$ cation 


\section{S-19}

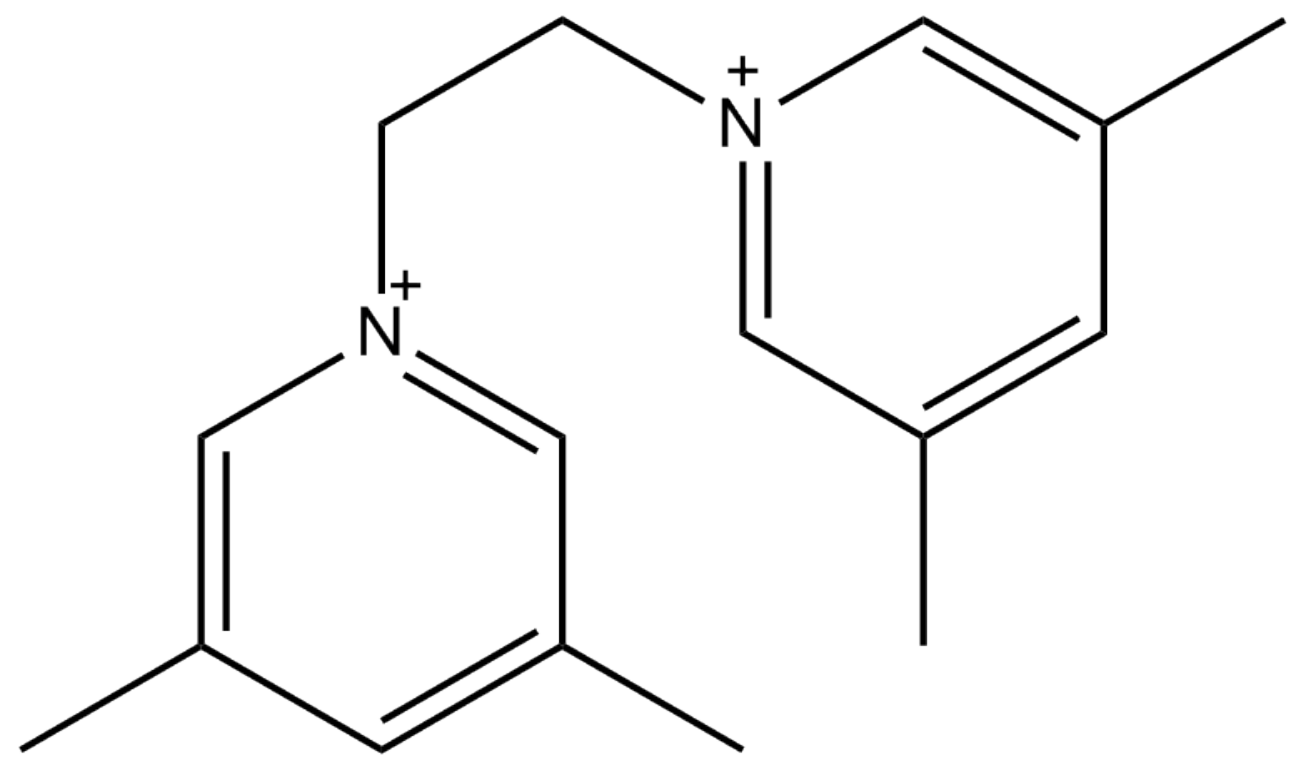

Figure S14. Structure of $(3,5-\mathrm{MePy}) \mathrm{C}_{2}{ }^{2+}$ cation

Thermogravimetric analyses (TGA) were carried out on a TG 209 F1 Iris thermobalance (NETZSCH, Germany). The measurements were made in a helium flow in the temperature range of $30-450^{\circ} \mathrm{C}$ using the heating rate of $10^{\circ} \mathrm{C} \mathrm{min}^{-1}$ the gas flow rate of $60 \mathrm{~mL} \mathrm{~min}^{-1}$ and open $\mathrm{Al}$ crucibles.

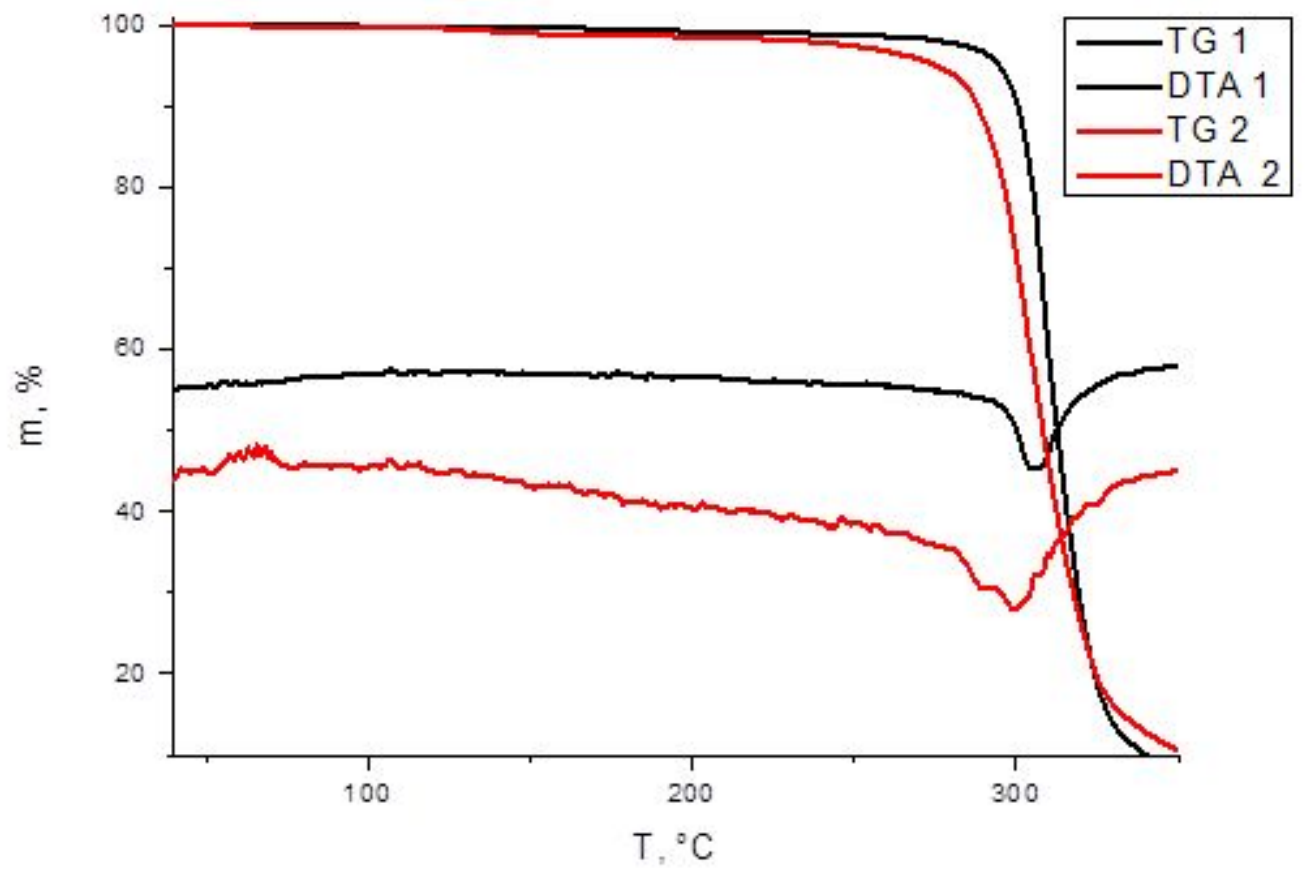

Figure S15. TGA curves for 1 and 2 